\title{
Development of Muscle-Power Measuring and Training System by Utilizing Electric Dynamometer for Aged Senior*
}

\author{
Kiyoshi YANAGIHARA**, Katsuyuki RYU**, \\ Tokuma MIHARA** and Kensuke TSUCHIYA*** \\ ${ }^{* *}$ Ariake National College of Technology, Dept. of Mechanical Engineering \\ Higashi-hagio-cho 150, Omuta-shi, Fukuoka, 836-8585 Japan \\ E-mail: kiyoshi@ariake-nct.ac.jp \\ *** Institute of Industrial Science, The University of Tokyo \\ Komaba 4-6-1, Meguro-ku, Tokyo, Japan
}

\begin{abstract}
New measuring and training method of muscle power for senior age, that utilizes small 'electric generator' instead of conventional 'spring force', is described in this paper. Our study clarified that this method can measure not only the muscle power, but also the reflection time and the muscle strength without any physical stress on aged senior subject. Meanwhile, another advantage of employing electric generator is that generator can electrically produce regeneration resistance as the load for rehabilitation. This paper also describes whether the developed system can be applied for rehabilitation. With a view of physiotherapist, various investigations were executed. The evidences show the effects of the developed training method and the developed system.
\end{abstract}

Key words: Measurement, Medical and Welfare Assistance, Human Engineering, Sensor, Control Device

\section{Introduction}

The ratio of aged senior in Omuta, that author's institute locates in, is increasing. Currently it is nearly $30 \%$, one of the highest levels in Japan. Therefore to guarantee senior's healthy retired life is an important issue for the local government. Thus one of the important missions is to understand their physical health.

Generally, in order to evaluate physical performance, Physical Measurement is adopted in Japan. According to "New Physical Measurement"(1) which is edited by Japanese Ministry of Education, Culture, Sports, and Science and Technology, Activity of Daily living (ADL) screening is utilized to evaluate the physical performance of senior who is over age-65. In this screening, grasping-force measurement is also utilized as a criteria to evaluate of muscle performance since muscle performances (Maximum Muscle Force) of a person's whole body is in proportion to his grasping-force statistically.

Currently Smedley type measuring instrument is used to measure grasping-force. Because it is economy and easy to use. This instrument, however, is based on Hook's law to measure the grasping-force. Therefore in the case to apply it for senior, Senior can tend to do bearing-down action that a person tries to perform his maximum muscle-power with his extreme muscle-tension as possible. This can lead the subject to be under physically stressful and dangerous situation. 
Therefore this paper clarifies physical stress of subject when Smedley type dynamo is applied to senior to measure grasping-force at first. Then the new measuring method and system for senior that employs small dynamo is proposed, and we clarify whether it can provide the stress-free measuring for senior or not.

Meanwhile using dynamo suggests the system provide not only measuring function but also training function by utilizing employing regeneration characteristic of dynamo as motor. Therefore the training method by regeneration is also proposed, and the effect of training method is discussed.

\section{Stress on subject while measuring grasping-force}

Figure 1 shows that physical stress on senior subject when grasping-force is measured with Smedley type dynamo. It shows blood-pressure change before and after the grasping-force of 80-years-old male and 38-years-old subject was measured respectively. The blood-pressure of 80 subject rises after grasping-force measuring, although that of age 38 subject is same level. This demonstrates that stress to perform maximum muscle force of age 80 subject is kept after grasping-force measurement. Therefore in order to support health-cares of seniors, stress-free measuring with lighter load as possible should be needed. In this study, in order to overcome the above circumstances, referring a mechanism of chassis dynamometer, newly developed grasping-power measurement by utilizing small dynamo is proposed.

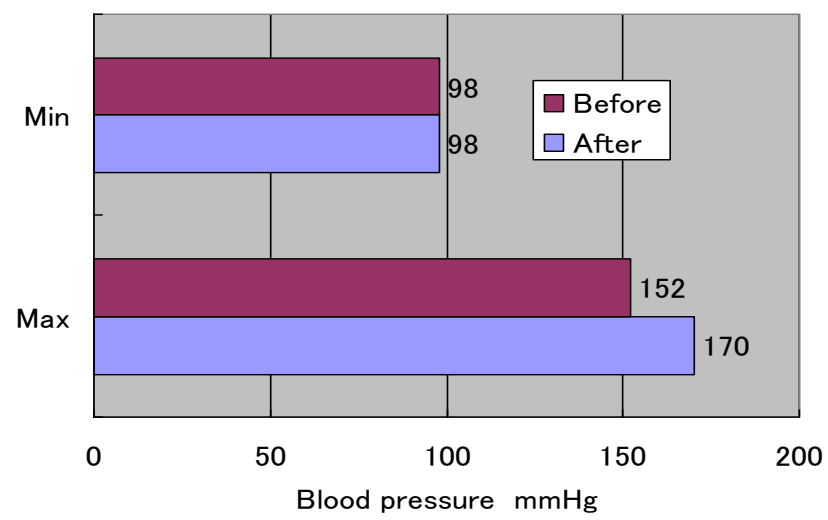

(a) male age- 80

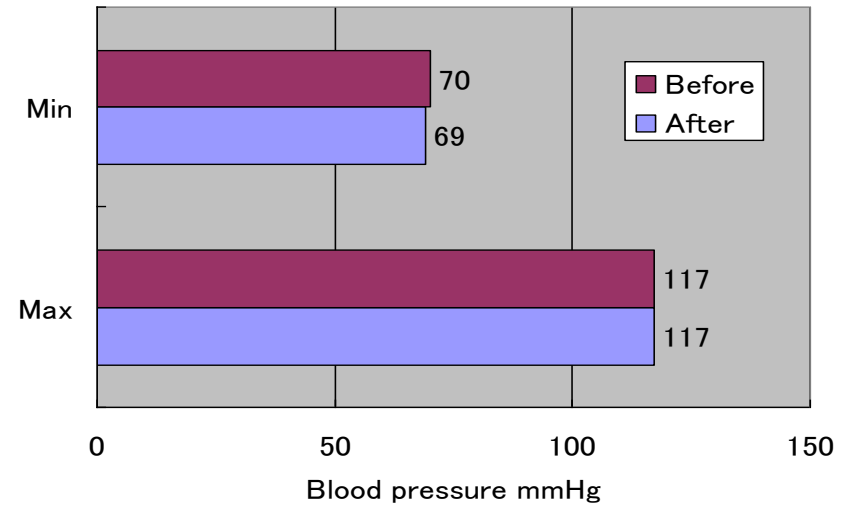

(b) male age-38

Fig. 1 Blood-pressure changes in grasping-force measured by using Smedley type dynamo. 


\section{Proposal of grasping-power measuring system by using small dynamo}

\section{$3 \cdot 1$ measuring system}

Figure 2 shows the proposed measuring system. The input-device of the system is a small dynamo for charging a mobile-phone battery, called Hand-held Dynamometer which is made by William telecom Co. Itd. It is modified not to charge the mobile-phone battery but to output voltage for the measuring purpose. The dynamometer has a lever whose stroke is $20 \mathrm{~mm}$. A subject holds this lever as shown in Fig. 2, then if he/her strokes the lever as fast as possible after Personal Computer (PC) display of the system indicates a start of measuring, a lack and a pinion transmit the power which the subject generates to a small motor in the input-device. The power which the subject inputs rotates a rotor whose moment of inertia is $3 \cdot 10^{-4} \mathrm{Nm}$, voltage in proportion to the rotation speed is produced.

When grasping-force $F$ strokes the lever with velocity $V$, if the power $P$ rotates the rotor of moment of inertia $I$ with torque $T$ and angular velocity $\omega$, the following relation is derived from them:

$$
P=T \cdot \omega=I \cdot \omega \cdot(d \omega / d t) \quad=F \cdot V
$$

Thus by using dynamometer system, voltage change which is in proportion to muscle power to grasp an object is obtained. The obtained voltage is recorded by PC and the data is analyzed. The experimental data is collected from 7 subjects which are consist of men and women from age-5 to age- 80 .

Physically Power is Force $\times$ velocity. Conventional Smedley type dynamometer records "Force". The proposed dynamo system, however, records "power". Therefore we define a new criterion as "grasping power" instead of "grasping-force" in this paper. Minimum grasping-force to stroke the lever of the input-device is $5.49[\mathrm{~N}]$. This is too low level to increase a blood-pressure of a subject. Meanwhile, in order to make a precise measurement, a subject must stroke the lever of input-device as fast as he/she can. Because if measurement is finished in very short time with subject's maximum velocity of their muscle shrinking, it is principally considered that the Force: $\mathrm{F}$ is constant, and $\mathrm{F}$ is estimated from the output power of device.
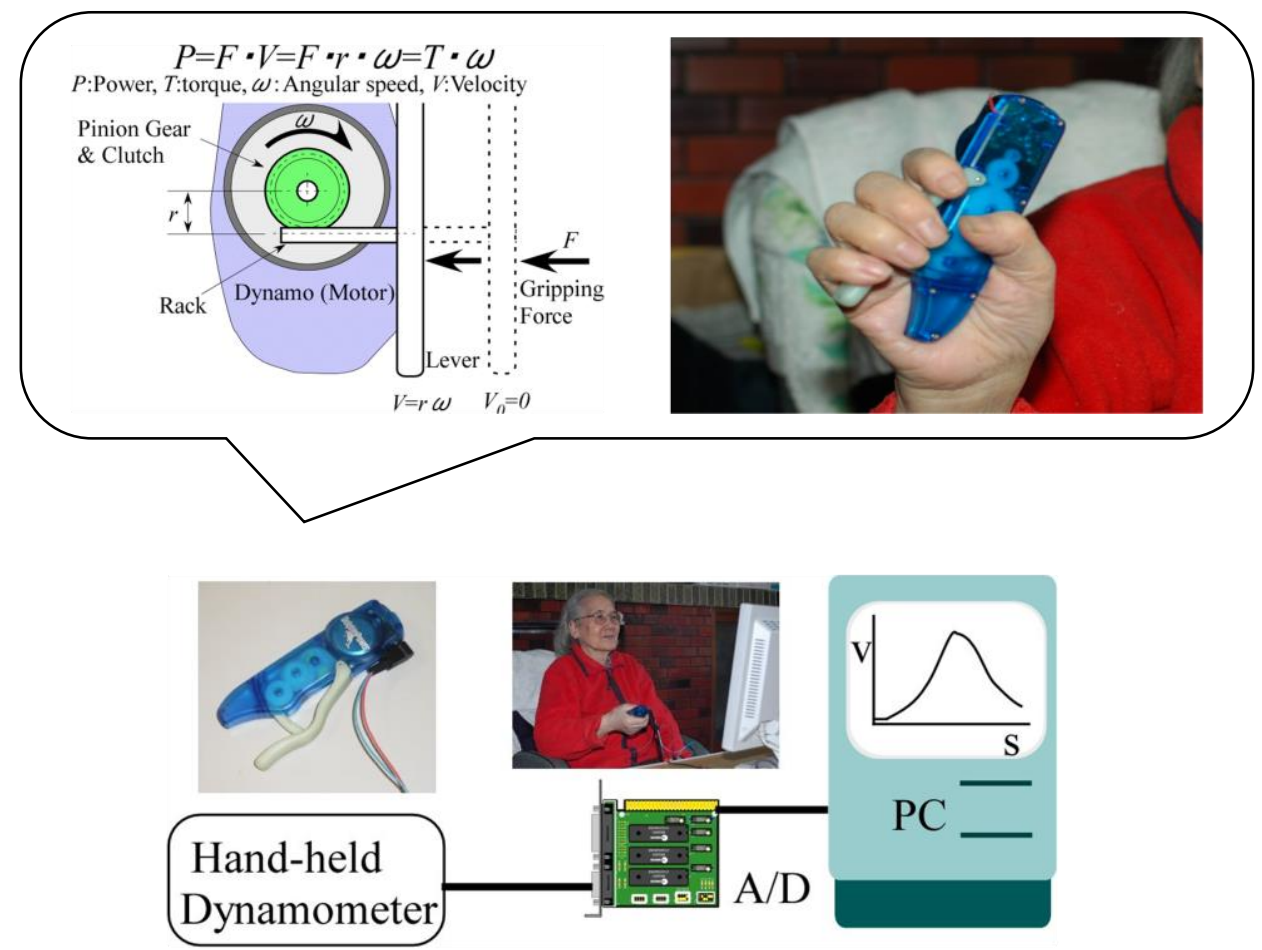

Fig.2 Measuring system 


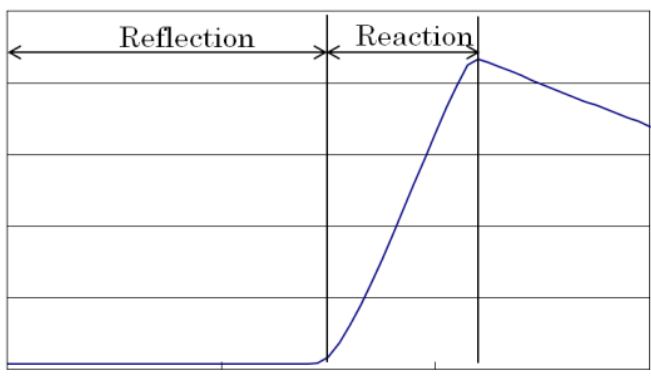

0.2

0.4

0.6

Time $\mathrm{s}$

Fig.3 Measured voltage (female Age-75 subject)

\section{$3 \cdot 2$ experimental results}

Figure 3 shows a grasping-force obtained as a change of voltage. The time $0 \mathrm{~s}$ is the start of measuring. From $0 \mathrm{~s}$ to $0.3 \mathrm{~s}$, the voltage is constant at about 0 [V]. This region means the time for the subject to reflex. After $0.3 \mathrm{~s}$ of the reflex time, the voltage is rising rapidly. Corresponding to muscle power of the subject, a rotor of the dynamo is accelerated. Therefore a steep rise of the output voltage in proportion to the acceleration is obtained as shown in the figure.

When the subject strokes the input lever to the stroke-end, a clutch separates the motor from the transmission. Moment of inertia of rotor, however, stores energy. It can rotate the rotor, and voltage is still generated. Therefor the voltage is gradually decreasing after the clutch works, as shown from $0.4 \mathrm{~s}$ to $0.6 \mathrm{~s}$ of fig. 3 .

Figure 4 shows a correlation between the measured maximum voltage and grasping-force obtained with Smedley dynamo. Grasping-force of each subject can be estimated from the maximum voltage which grasping power of each subject generates, since the figure demonstrates good proportion between them.

Meanwhile, transition of voltage in Fig. 3 shows change of muscle power. The transition shows velocity transition of muscle shrinking if the load in stroking the lever is constant. Therefore if trend of rising voltage from ground level to maximum is steep, it also means that acceleration of shrinking of muscles is fast, it shows that the subject can hold any objects surrounding him/her to keep his/her position in an urgent situation.

Figure 5 shows differential coefficients of rising trend of the voltage from age- 5 to age-75 female subjects. In this figure, numbers of labels show the ages of each subject, letter "F" also means female. Their grasping powers of both left and right hand are measured and the differential coefficients are calculated in each hand as shown in the figure. The result shows that grasping-forces of age-75 right and age-11 left are almost the same. The weight of age-75, age-11, however, is different, 490[N] and 290[N], respectively. It indicates that age-75 is more difficult to prevent falling to ground while catching and holding hand-rail and so on, in urgent situation than age-11. Because grasping power can be less to keep subject falling down momentum. 


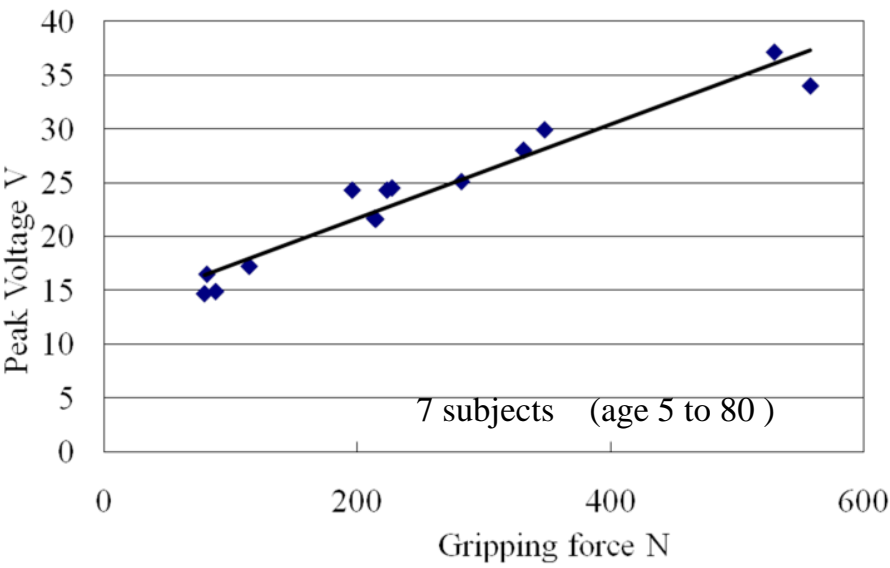

Fig.4 Gripping force V.S. Peak Voltage

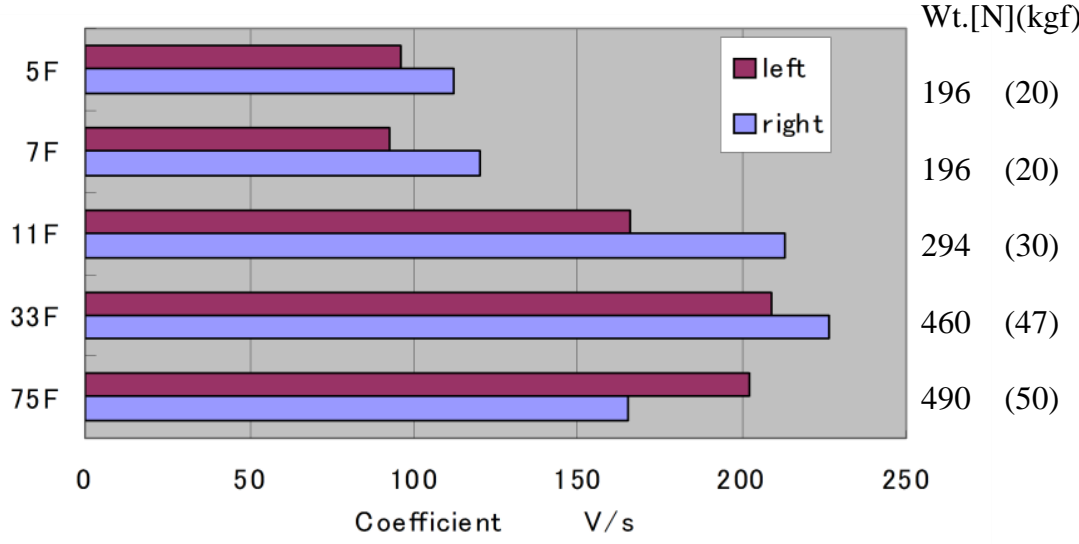

Fig.5 Differential coefficient of measured voltage

\subsection{Verification of safety of proposed measuring-method}

Finally it is confirmed how the proposed measuring method can affect seniors. Figure 6 shows blood-pressure changes of age-75 female and age- 85 male subjects before and after grasping power measurement. Each blood-pressure keeps almost the same value. Therefore the results prove that the proposed measuring method and system are safety for senior. 


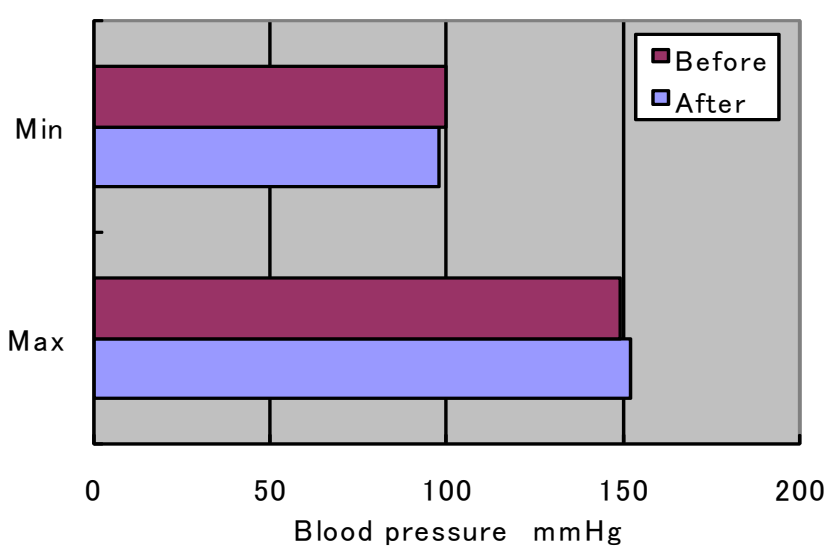

(a) female age-75

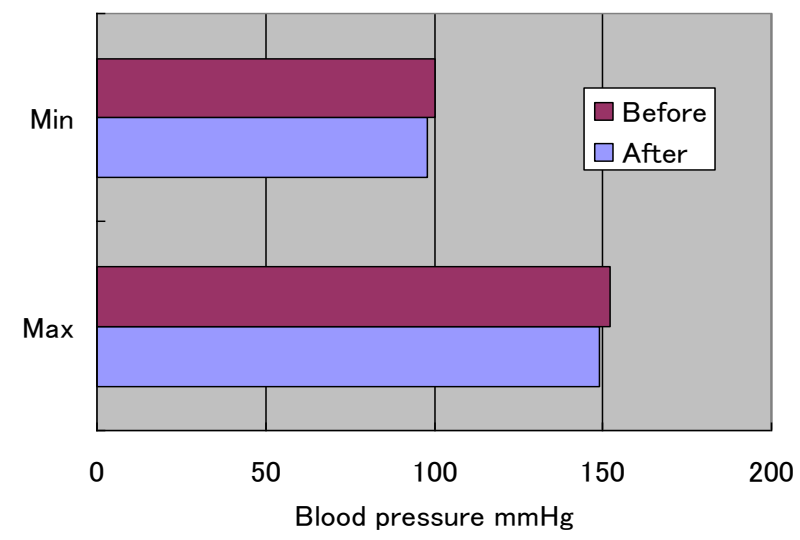

(b) male age- 80

Fig. 6 Blood-pressure changes in grasping-force measured by using newly developed hand-held dynamo.

\section{Development of training-mode function by utilizing regeneration-resistance}

\section{1 Confirmation of regeneration-resistance and lever load}

Dynamo can generate regeneration resistance when it generates electricity. If the control of regeneration-resistance is available, the one system can provide not only measuring function, but also training function simultaneously, while referring muscle-performance of each subject. In order to realize the training function, we need to confirm how much load of a lever-stroke can be produced by regeneration of dynamo.

Figure 7 shows the experimental set-up to measure the lever-load produced by regeneration-resistance. In this experiment, regeneration-resistance is measured by pulling pull-tension gage quasi-stastically while the fixing input-device with a jig.

Figure 8 shows lever-stroke resistance V.S. circuit-resistance of dynamo. The lever-stroke resistance is in inverse proportion to the circuit-resistance attached to dynamo. The lever-stroke resistance is $7.2[\mathrm{~N}]$ when the circuit of the dynamo is shorted. Then it gradually deceases. In case of $20[\Omega]$, the lever-stroke resistance is 5 [N ]. It indicates that the system provides an appropriate load to a subject immediately if an appropriate circuit-resistance is programed electrically while referring to muscle-performance of the subject.

Figure 9 shows developed grasping power measuring and training system for senior. The system is added the training function by regeneration of dynamo into measuring system 
of Fig. 1. A subject can select two modes which are consist of the measuring mode by measuring voltage of dynamo and the training mode by producing electrical load of regeneration. In following section, the effect of training mode is discussed.

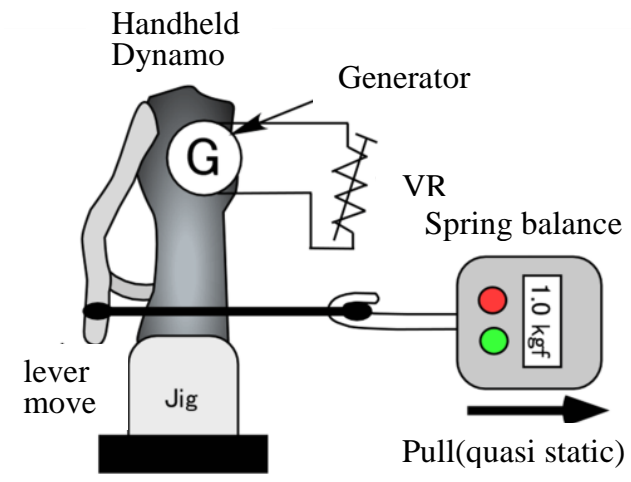

Fig. 7 Illustration of measurement of regeneration-resistance of generator

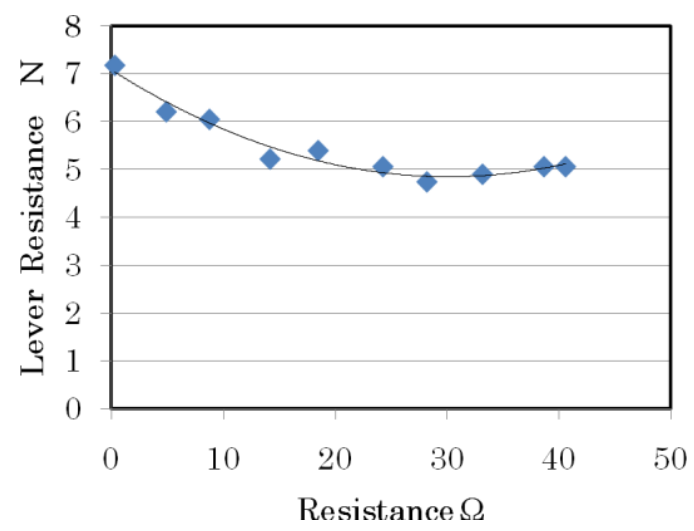

Fig.8 Lever-stroke resistance V.S. circuit resistance

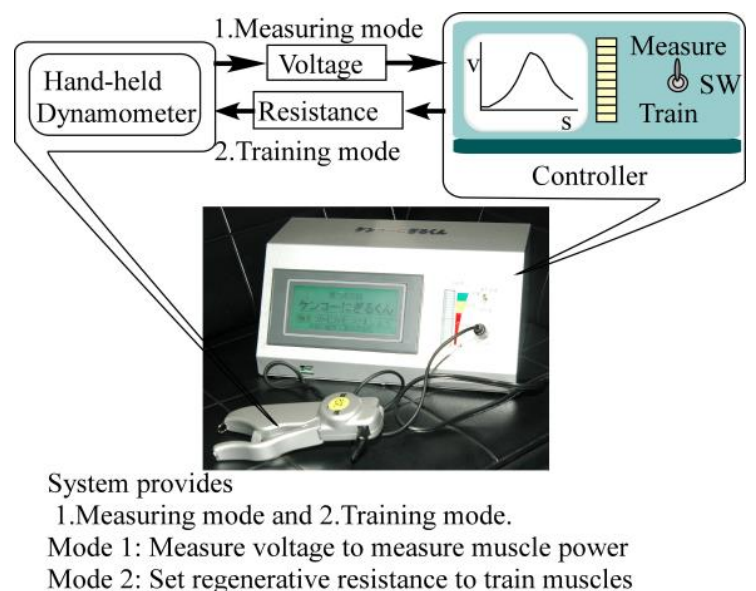

Fig.9 Illustration of developed system 


\section{2 effect of muscle-power improvement for senior}

In case of muscle training for senior, it is desired for senior to obtain continuous improvement while providing appropriate load. In "New physical measurement (1)", measuring-standard for a subject is that systolic blood-pressure is under $160 \mathrm{mmHg}$, diastolic blood-pressure is above $95 \mathrm{mmHg}$, heart rate is below $100 \mathrm{~min}^{-1}$. Based on the standard, training-load for senior to be applied is determined. After consideration with senior subjects, the following 4 load-conditions; "Normal" ( $\infty \Omega)$, "Heavy" $(19 \Omega)$, "Heavier " $(8.5 \Omega)$, "Heaviest" $(0 \Omega)$, is programed while referring the result of Fig.8.

A subject selects these four modes while referring his/her physical condition and feeling. He/She trains instantaneous muscle-power with speedy lever-strokes of the input-device. In the selection of the lever-stroke load, firstly subject must select as strong as possible, then he/she can chose lighter load whenever he/she feels hardness physically. According to the principle, the numbers of lever-strokes are counted, and the training effect is discussed. For the experiment to find the effects, age- 59 female and age- 81 female are selected, and 28 days training-experiment is executed.

Figure 10 shows the lever-strokes of left-hand of age- 81 female-subject. Systolic blood-pressure of age-59, age-81 subject is $110,118 \mathrm{mmhg}$ respectively when they lie quiet in bed. The experiment is executed after it was confirmation that their selected load of one lever-stroke does not increase their blood-pressure. In this figure, the front series of data shows selected "Heaviest" of each day. Along to backward series of data, "Heavier", "Heavy", "Normal" data series is shown respectively. Total-strokes per day is shown as "Total". The result demonstrates that lever-strokes per day increasing every day. The increase is observed in each load. It is possible that the increase of lever-stroke indicates the increase of not only muscle power but also muscle force and stamina of subject.

Some studies ${ }^{(1),(2),(3)}$ report that subject can improve maximum power if he/she exercises under his/her consideration that he/she does it as speedy, or as more pitches as possible. Therefore the increase of lever-stroke to improve muscle-power in previous section may also provide increase muscle-force. Thus in order to confirm the hypothesis, gripping-force of each subject is also measured with Smedley dynamometer every week after the beginning of their experiment, as shown in Fig. 11. The figure shows the gripping force of not only age- 81 female-subject but also age-59 subjects for comparisons. Although the difference between right and left hand, the gripping forces of both subjects are improved rapidly after one week from the beginning of the training. Then they increase gradually.

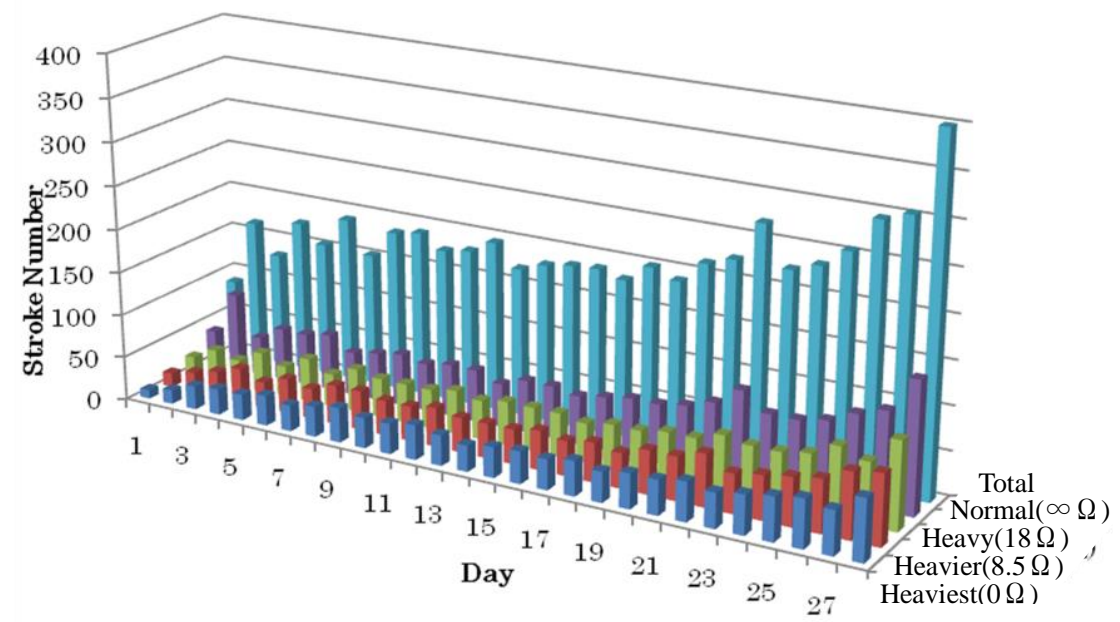

Fig.10 Lever-strokes per day of age-81 female subject in grasping training of left hand 


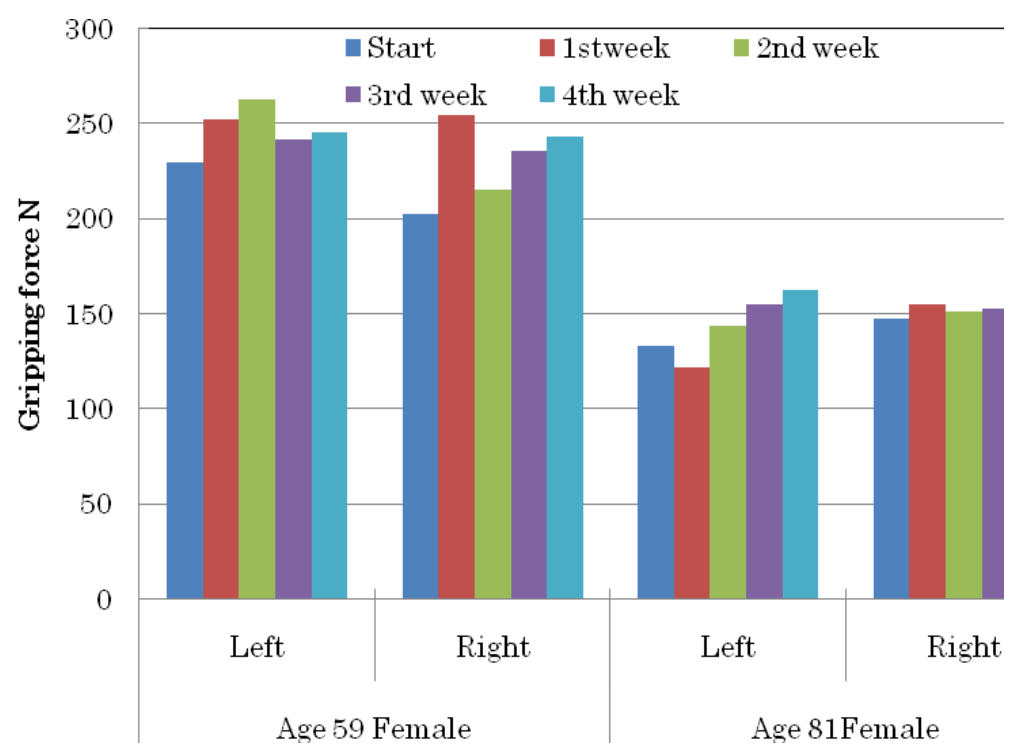

Fig.11 Gripping-force changes every week from the start of this experiment (measured with Smedley dynamo.)

\section{$4 \cdot 3$ application to rehabilitation}

The new training-method is applied to age- 83 male-patient of Cubital Tunnel Syndrome. The patient had light paralysis about the 4 th and the 5th finger of his right hand in summer of 2008. Then he was operated in hospital.

Figure 12 shows Lever-strokes per day of 83 years old male subject in grasping rehabilitation of right hand, as well as Fig. 10. Generally appropriate physical exercises enhance recovery of a patient ${ }^{(6),(7)}$. The results show that "Total" lever-stroke per day is increasing day by day, the other load-settings are increasing too. In this experiment, grasping-force is recorded every week as well as Fig. 11. Although his grasping-force is decrease just after operation on the $23 \mathrm{rd}$ of Jul, it seems that the proposed method enhances the recovery.

In order to confirm the effect of the proposed rehabilitation, cross-sectional photos with Magnetic Resonance Imaging (MRI) are taken as shown in Fig. 12. Figure 14 (a), (b) shows the image after 20 days past from the operation, that after 2 months, respectively. The MRI images are taken in the place which is the view of $10 \mathrm{~cm}$ from the elbow to the wrist. The enclosed cross-sectional areas with green lines are muscles which work for grasping. The images demonstrate that the area increased and effect of the proposed rehabilitation. 


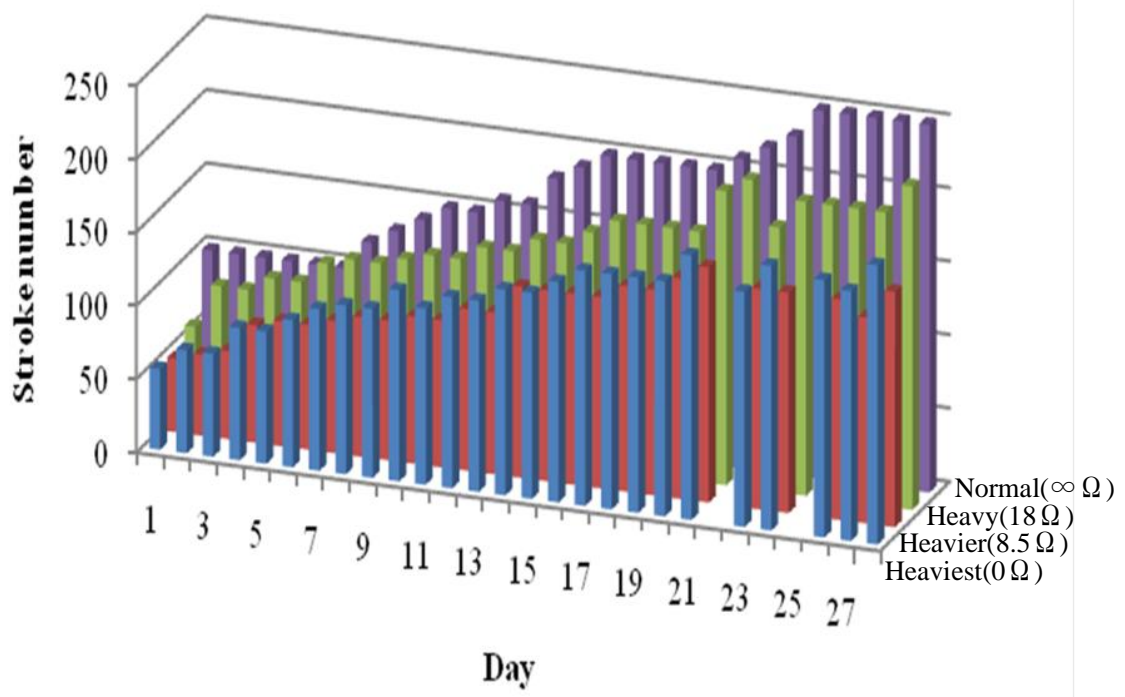

Fig.12 Lever-strokes per day of 83 years old male subject in grasping rehabilitation of right hand

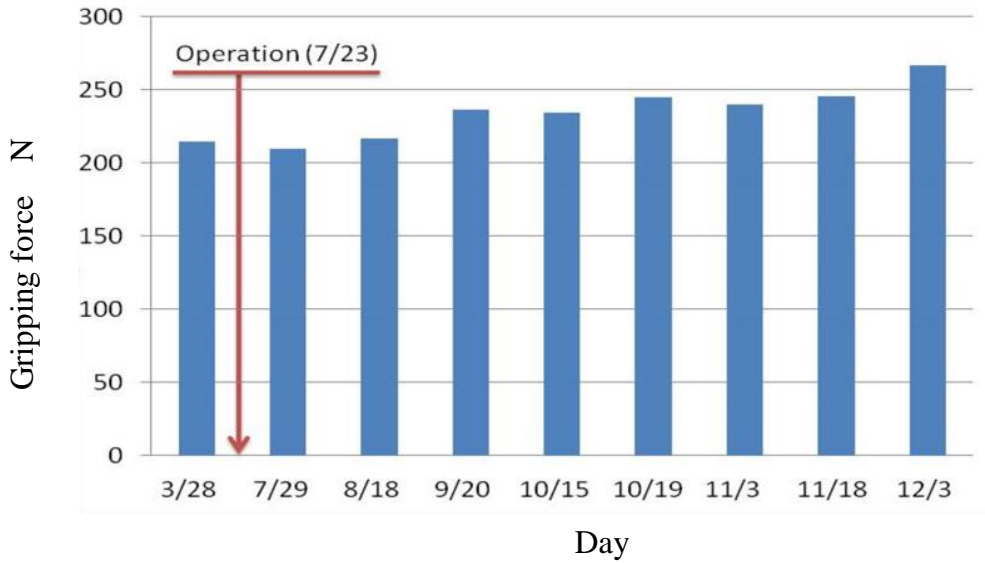

Fig.13 Gripping force of left hand of 83 years old male subject per day by Smedley type dynamometer 


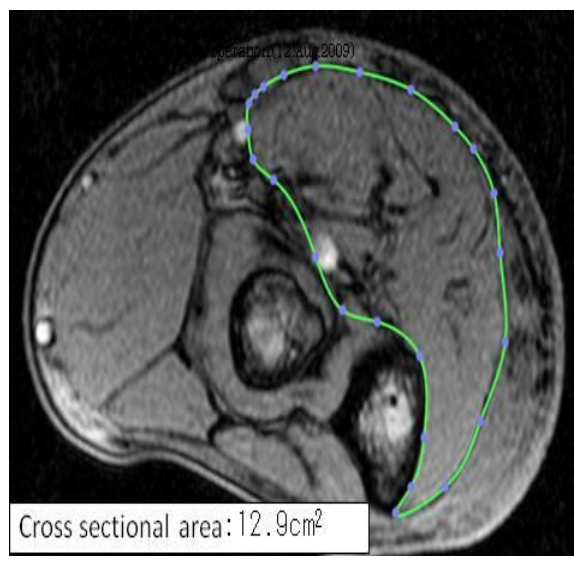

(a)20 days after operation ('09 Aug. 12)

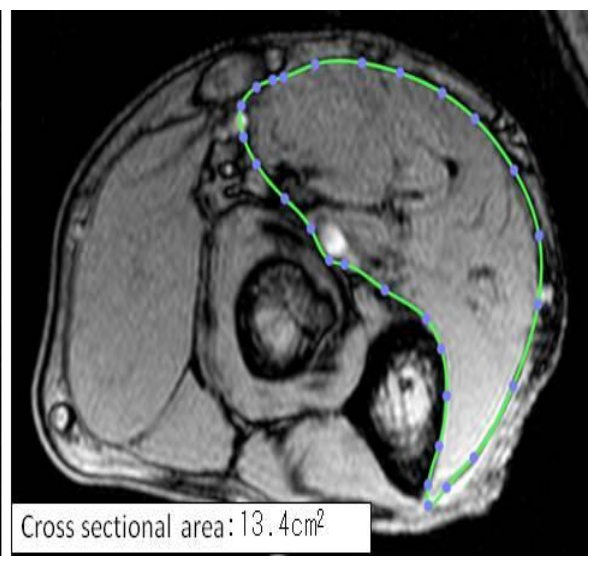

(b) 2 months with rehabilitation ('09 Oct. 14)

Fig.14 MRI Images before and after rehabilitation from operation

\section{Conclusions}

New physical stress-free measuring and training method of muscle power for senior is proposed in this study. The method employs electric generator, and new measuring and training system with the method also developed. The obtained results are following.

(1) The proposed muscle-power measuring method that employs a small generator is less physical stress than the conventional muscle-force measuring system that is based on Hook's law. Because no bearing-down action is required for seniors in the new method.

(2) Output voltage that is measured by hand-held dynamometer: muscle-power, demonstrates linear correlation with muscle force: grasping-force.

(3) Not only measuring peak muscle-force but also observing dynamical muscle-power is available with the proposed measuring method.

(4) By means of attaching electric-circuit resistance from 0 to $20[\Omega]$ to hand-held dynamometer, regenerating resistance for training can be produce from 5 to 7.2 [N].

(5) Long-term trainings of senior subject are executed by using the load of the above mentioned (4). Improvement of muscle-force is confirmed with increase of lever-strokes, grasping-force, and muscle area that is clarified with MRI observation.

\section{Acknowledgement}

The cooperation of Kazuhira Sakamoto, Yabegawa electric Co. Ltd., for the production of the training system, and suggestions of Katsuki Ego, Omuta city hospital, for designing the training-program from the view of physical science, and cooperation of the group-home Fureai for measuring grasping powers of seniors, are gratefully acknowledged.

This work was supported by JSPS KAKENHI Grant Number 2050499. 


\section{References}

(1) Ministry of Education, Culture, Sports, Science and Technology, New Physical Measurement, Ver.4(2006), Gyousei. (in Japanese)

(2) Kanehisa,H., Muscle power and Training, Science of Physical Education, Vol. 39, No. 4 (1989), pp. 274-285.(in Japanese)

(3) Jonsson, B., Biomechanics X-B (1987), pp.615-620, Human Kinetic Publisher.

(4) Kaneko, K., Fuchimoto, T., Tashiro, H., Suei, K., Training effect on force, speed, and power of human muscle, Physical Strength Science, Vol. 30 (1981), pp.86-93.(in Japanese)

(5) Hellenbrant, F.A., Houz, S.J., Methods of muscle training, The influence of pacing Phys. Ther. Rev., Vol. 38 (1958), pp. 319-322.

(6) The Japan Society of Mechanical Engineers ed., JSME Mechanical Engineering Handbook applications y 9: Medical, Welfare, and Bio-Systems-,(2008), p. 218, he Japan Society of Mechanical Engineers.(in Japanese)

(7) Sugi, H., Sports Phisiology, Ver.3(2003), p. 57, Nankodo.(in Japanese) 